\title{
Conductivity of a two-phase composite: An approach based on bounds
}

\author{
A.V. Goncharenko \\ Institute of Semiconductor Physics, NAS of Ukraine, \\ 45, prospect Nauki, Kyiv, 03028, Ukraine, E-mail: avg@isp.kiev.ua
}

\begin{abstract}
Using the theory on the bounds, a general representation for effective conductivity of a twophase composite is proposed. The representation contains two parameters that depend on the composite topology and can be determined from experimental data.
\end{abstract}

Keywords: composite, effective conductivity, Bergman representation.

Paper received 29.09.99; revised manuscript received 15.12.99; accepted for publication 17.12.99.

Nowadays it may be considered as well established that the problem how to determine the effective (macroscopic) conductivity (as well as other effective coefficients, such as permittivity, permeability, heat conductivity, diffusion coefficient) for a composite system can be reduced, effectively, to determination of some topological characteristics of this system. The beforesaid may be clearly demonstrated especially using a formalism proposed by Bergman $[1,2]$. In the Bergman representation the effective conductivity of a twophase composite, $\sigma$, is to obey the following equation:

$$
F(s)=p_{1} \sum_{n} \frac{B_{n}}{s-s_{n}} .
$$

Here, $F=1-\tilde{\sigma} / \sigma_{2}, s=\left(1-\sigma_{1} / \sigma_{2}\right)^{-1}, \sigma_{1}, \sigma_{2}$ are the conductivities of phase 1 and 2 , respectively; $p_{1}$ is the volume fraction of phase 1 . The poles $s=s_{n}$ correspond to the resonant frequencies of normal (surface or interfacial) modes of the electric field in a system; $s_{n}$ are the so-called depolarization factors. $B_{n}$ give the mode strength values. In this case both $B_{n}$ and $s_{n}$ depend on the composite topology only. So the expression (1) makes it possible to separate dependence of $\sigma$ upon the phase conductivities, on one hand, and upon the composite topology, on the other hand. Owing to the topological (configurational) fluctuations all the poles in the expression (1) (with one exception - the pole at $s=0)$ are, generally speaking, broadened. That is why, instead of (1), its generalization in the form of an integral representation

$F(s)=p_{1}\left(\frac{A}{s}+\int_{0}^{1} \frac{g(t)}{s-t} d t\right)$

is often used $[3,4]$. Here the pole at $s=0$ is set off; the necessary topological information is contained in the reso- nance spectral density function $g(t)$. In other words, this function gives, in a physical sense, a distribution of depolarization factors. This distribution is related to the geometric shape (topology) of the regions occupied with phase 1 , or to the surface mode spectrum. The coefficient $A$ (also known as the percolation strength) determines if percolation occurs in the system. One can show (see, e.g., [5]) that $p_{1} A=\tilde{\sigma} / \sigma_{1}$. Hence if phase 1 is a conducting one, then the condition $A>0$ means that the system is over the percolation threshold. In this case $A$ may be considered as a phase 1 fraction that contributes to the dc conductivity [5]. The representation (2) is supplemented also with the following relationships known as the sum rules [4]:

$$
\begin{aligned}
& \int_{0}^{1} g(t) d t=1-A \\
& \text { and } \\
& \int_{0}^{1} \operatorname{tg}(t) d t=\frac{1}{3}\left(1-p_{1}\right)=\frac{p_{2}}{3} .
\end{aligned}
$$

The expressions (1) and (2) have often been used in actual practice (see, e.g., [6-12]). However, their wide use involves a number of problems. The most essential of them is that, broadly speaking, a general form of the function $g(t)$ is unknown. Of course, one may determine this function from an experiment, for example, from the reflection spectra; the corresponding technique is discussed in [13]. Such a determination, however, imposes heavy demands on experimental data that not necessarily can be met.

The following spectral density representation basing on general grounds concerning a behavior of the dielectric response function for brine-saturated rocks was pro- 


\section{A.V. Goncharenko: Conductivity of a two-phase composite: An approach...}

posed in [4]:

$$
g(t)=C t^{-b}(1-t)^{e} .
$$

Here the coefficient $C$ may be found from [3] or [4], and only one of the exponents $b$ and $e$ is independent. Thus the representation (5) is actually one-parameter. Ghosh and Fuchs [14] have proposed the following generalization of (5):

$$
g(t)=\left\{\begin{array}{l}
\frac{C}{t}\left(t-t_{2 L}\right)^{1-\alpha}\left(t_{2 U}-t\right)^{\beta} \\
0 \leq t_{2 L} \leq t \leq t_{2 U} \leq 1, \alpha>0 \\
0, \text { beyond the interval }
\end{array}\right.
$$

This representation introduces new parameters $\left(t_{2 L}\right.$ and $\left.t_{2 U}\right)$. It is three-parameter since it contains three independent phenomenological quantities. They are topology-dependent and can be found from experiment only. (Of five parameters that enter the representation (6) two ones are not independent because they may be determined using the sum rules.) It should be noted, however, that the representation (6), as well as (5), is based on some intuitive considerations rather than on strict theoretical grounds.

In [15] an interpolation scheme has been proposed. In it the effective permittivity of a binary composite at an arbitrary $p$ may be represented as a result of interpolation between the two extreme points corresponding to $p \rightarrow 0$ and $p \rightarrow 1$. Later it was shown [16] that the final expression may be also obtained as a result of a linear interpolation of the Bergman spectral function between two its bounds that correspond to the limits of extremely low and extremely high concentration of one of phases. Certainly, these results are of limited utility because only one specific composite topology is actually considered. However, they lead to a rather apparent idea: to obtain a general expression for $\sigma$ using interpolation of bounds plus introduction of some additional topology-dependent parameters.

Here we propose another way of determining the general form of function $g(t)$ (and, as a result, also $\sigma$ ). This approach is an immediate consequence of the theory on bounds for conductivity (permittivity) of a composite [1720]. Its idea is very simple. It is parametric setting of a domain of all possible (allowed) $\sigma$ values when its boundary values are set.

Let us consider two pairs of bounds that are of most interest. The first pair (for real values of $\sigma_{1}$ and $\sigma_{2}$ ) has been proposed in [17] as a result of applying the variational approach. (It should be noted that these bounds may be also obtained from the spectral representation (1) [1,2] if $B_{n}$ and $s_{n}$ are treated as free parameters and the function $F$ extrema are sought.) Later in [18-20] an interpretation of these bounds in the complex plane $\operatorname{Re} F$-Im $F$ has been given, as well as some new bounds have been proposed. If the conductivities $\sigma_{1}, \sigma_{2}$ and filling factors $p_{1}, p_{2}$ for both phases are known, then the effective conductivity of a composite has to lie in a domain bounded with two arcs (bounds). In the complex plane $\operatorname{Re} F$-Im $F$ these arcs are set by the following parametric equations:

$$
F_{1}^{(1)}(s)=\frac{p_{1}}{s-s_{1}}
$$

and

$F_{1}^{(2)}(s)=\frac{p_{1} s-s_{2}+p_{2}}{s\left(s-s_{2}\right)}$,

where the real parameters $s_{1}$ and $s_{2}$ are to meet the inequalities $0 \leq s_{1} \leq p_{2}, p_{2} \leq s_{2} \leq 1$. The view of $F_{1}^{(1)}$ and $F_{1}^{(2)}$ bounds in the complex plane is shown in Fig. 1. It should be noted that the point $A$ corresponds to the condition $s_{1}=0$, while the point $B$ corresponds to the condition $s_{1}=p_{2}$. Then the movement from point $A$ to point $B$ along the bound $F_{1}^{(1)}$ corresponds to $s_{1}$ changing from 0 to $p_{2}$, while that from point $B$ to point $A$ along the bound $F_{1}^{(2)}$ corresponds to $s_{2}$ changing from $p_{2}$ to 1 .

Another pair of bounds can be obtained under an additional requirement that a composite is either isotropic or of cubic symmetry $[18,20]$. In the complex plane these bounds also are arcs that are set with the following equations:

$$
F_{2}^{(1)}(s)=\frac{p_{1}}{s} \frac{s-s_{3}}{s-s_{3}-p_{2} / 3}
$$

and

$$
F_{2}^{(2)}(s)=p_{1} \frac{s-s_{4}}{\left(s-s_{4}\right)\left(s-p_{2} / 3\right)-2 p_{2}\left(1-s_{4}\right) / 3} .
$$

In this case the point $C$ corresponds to the condition $s_{3}=0$, while the point $D$ corresponds to the condition $s_{3}=2 / 3$. Then the movement from point $C$ to point $D$ along the arc $F_{2}^{(1)}$ corresponds to the $s_{3}$ changing from 0 to $2 / 3$, while that from point $C$ to point $D$ along the arc $F_{2}^{(2)}$ corresponds to the $s_{4}$ changing from $2 / 3$ to 1 .

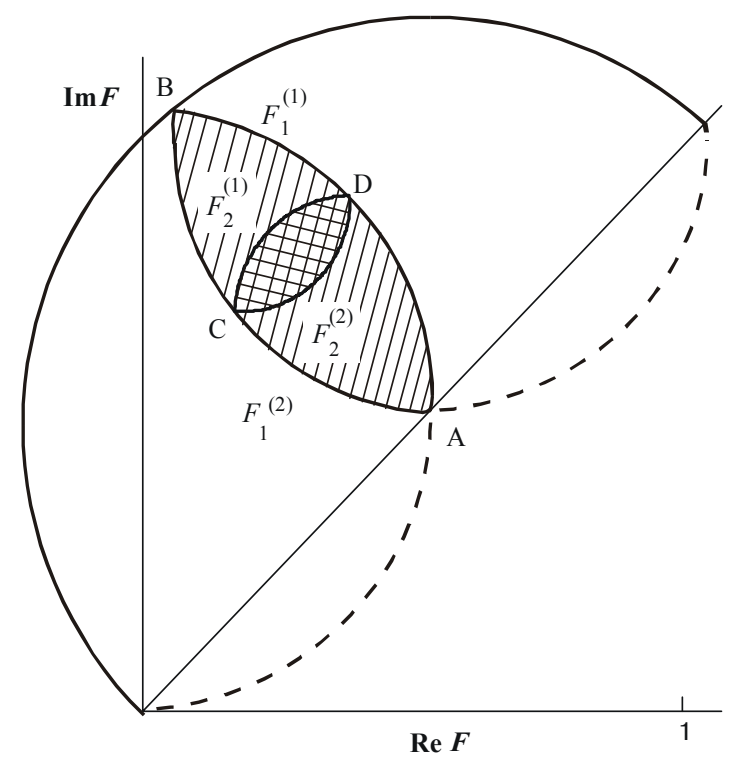

Fig. 1. Graphical display of bounds and allowed values regions for effective conductivity in the complex plane $F$. 


\section{A.V. Goncharenko: Conductivity of a two-phase composite: An approach...}

Now let us try to find equations that any point lying in a lune between $F_{1}^{(1)}$ and $F_{1}^{(2)}$, as well as between $F_{2}^{(1)}$ and $F_{2}^{(2)}$ (i.e., within the domain of allowed $\sigma$ values) has to obey. It is obvious that there are many solutions to this problem. We would like to demonstrate how one can find such equations using a rather evident fact: any point lying on a line segment connecting a pair of the corresponding bounds belongs to the above domain. (This statement is a consequence of the convexity of bounds.) This means that the region bounded with the pair of arcs (1)-(2) can be represented through the following linear combination:

$F_{1}(s)=r_{1} F_{1}^{(1)}(s)+\left(1-r_{1}\right) F_{1}^{(2)}(s)$.

Here the weight parameter $r_{1}$ sets a proximity to the left (or right) boundary. It is obvious that in this case the inequality $0 \leq r_{1} \leq 1$ is to hold. On the other hand, to complete (cover) the region $F_{1}$ one has to made a $2 D$ scanning procedure. Let us introduce, instead of $s_{1}, s_{2}$ in the relations (7)-(8), the only parameter

$q_{1}=\frac{s_{1}}{p_{2}}=\frac{s_{2}-1}{p_{2}-1}$.

This parameter determines a proximity to the point $A$ or $B$. Then changing $s_{1}$ from 0 to $p_{2}$ and $s_{2}$ from 1 to $p_{2}$ (i.e., the movement from the point $A$ to the point $B$ ) will automatically correspond to $q_{1}$ changing from 0 to 1 . Thus the expression (11) gives a two-parameter representation for the first region of $\widetilde{\sigma}$ allowed values. The scanning parameters are $r_{1}$ and $q_{1}$, if one takes into account that $s_{1}=p_{2} q_{1}$ and $s_{2}=1+\left(p_{2}-1\right) q_{1}=1-p_{1} q_{1}$. It should be noted that both parameters, $r_{1}$ and $q_{1}$, have to lie within the interval $[0 ; 1]$. The equation (11) may be also written in the following form:

$$
\begin{aligned}
& F_{1}(s)=p_{1}\left[\frac{1-r_{1}}{1-p_{1} q_{1}}\left(\frac{1-q_{1}}{s}+\frac{p_{2} q_{1}}{s+p_{1} q_{1}-1}\right)+\frac{r_{1}}{s-p_{2} q_{1}}\right]= \\
& =p_{1} \sum_{n=0}^{2} \frac{B_{n}^{\prime}}{s-s_{n}^{\prime}} .
\end{aligned}
$$

Here

$$
\begin{aligned}
& B_{0}^{\prime}=\left(1-q_{1}\right) \frac{1-r_{1}}{1-p_{1} q_{1}}, \\
& B_{1}^{\prime}=p_{2} q_{1} \frac{1-r_{1}}{1-p_{1} q_{1}}, \\
& B_{2}^{\prime}=r_{1}, s_{0}^{\prime}=0, s_{1}^{\prime}=1-p_{1} q_{1}, s_{2}^{\prime}=p_{2} q_{1} .
\end{aligned}
$$

By analogy, for the second region of $\widetilde{\sigma}$ allowed values one may write a linear combination of $F_{2}^{(1)}$ and $F_{2}^{(2)}$ as

$$
F_{2}(s)=r_{2} F_{2}^{(1)}(s)+\left(1-r_{2}\right) F_{2}^{(2)}(s) .
$$

If a parameter $q_{2}$ is introduced as $q_{2}=\frac{3}{2} s_{3}=3\left(1-s_{4}\right)$,

then the equation (14) may be considered as a two-param- eter representation of the second region $\left(F_{2}\right)$ with parameters $r_{2}$ and $q_{2}$ where $s_{3}=\frac{2}{3} q_{2}$ and $s_{4}=1-\frac{1}{3} q_{2}$. In this case, when $q_{2}$ changes from 0 to 1 , then $s_{3}$ changes from 0 to $2 / 3$ and $s_{4}$ changes from 0 to $2 / 3$. This corresponds to the movement from the point $C$ to point $D$. Having rewritten the equation (14) in an explicit form through parameters $r_{2}$ and $q_{2}$, we obtain

$$
F_{2}=p_{1} \sum_{n=0}^{3} \frac{B_{n}^{\prime \prime}}{s-s_{n}^{\prime \prime}},
$$

where

$$
\begin{aligned}
& B_{0}^{\prime \prime}=\frac{2 r_{2} q_{2}}{2 q_{2}+p_{2}}, \\
& B_{1}^{\prime \prime}=\frac{r_{2} p_{2}}{2 q_{2}+p_{2}}, \\
& B_{3}^{\prime \prime}=\left(1-r_{2}\right) \times \\
& \frac{1}{2}\left(p_{1}-q_{2}\right)+3 \sqrt{\left(p_{2}+3-q_{2}\right)^{2}-12 p_{2}\left(1-q_{2}\right)} \\
& \times_{2}^{\prime \prime}=\left(1-r_{2}\right)\left(1-B_{3}^{\prime \prime}\right), \\
& s_{0}^{\prime \prime}=0, s_{1}^{\prime \prime}=\frac{1}{3}\left(2 q_{2}+p_{2}\right), \\
& s_{2}^{\prime \prime}=\frac{1}{6}\left[p_{2}+3-q_{2}+\sqrt{\left(p_{2}+3-q_{2}\right)^{2}-12 p_{2}\left(1-q_{2}\right)}\right], \\
& s_{3}^{\prime \prime}=\frac{1}{6}\left[p_{2}+3-q_{2}-\sqrt{\left(p_{2}+3-q_{2}\right)^{2}-12 p_{2}\left(1-q_{2}\right)}\right]
\end{aligned}
$$

The equation (13) (as also (7), (8) and (11)) is applicable to anysotropic systems. Generally speaking, it gives only one of the diagonal components of the effective conductivity tensor. One can see that each of these components may have three poles. The first pole (at $s=0$ ), as was stated before, corresponds to the so-called percolation mode and characterizes occurrence of percolation in a composite. Two other poles characterize intrinsic surface (configurational) modes of a system. It should be noted that the term of the type $\left(s-s_{0}\right)^{-1}$ gives (with some constant factor) the dipole polarizability for a spheroid whose depolarization factor is $s_{0}$ [21]. Therefore, one may state that for a two-phase composite the component of the effective conductivity tensor is the same as for a system of equally orientated noninteracting spheroids of three kinds (evidently, the electric field orientation along the spheroid rotation axis corresponds to the above component). As could be seen, for two of these spheroids the depolarization factors are not independent; they are related by the expression:

$s_{1}^{\prime}+\frac{p_{1}}{p_{2}} s_{2}^{\prime}=1$.

The spheroids of the third kind $\left(s_{0}^{\prime}=0\right)$ are prolate needles (infinitive cylinders). Their presence is an evidence that percolation through phase 1 occurs. Thus, consideration of an actual composite may be replaced by consideration of 


\section{A.V. Goncharenko: Conductivity of a two-phase composite: An approach...}

two (or three if percolation occurs) conventional polarizable objects of spheroidal shape. (It is pertinent to note that the well-known Bruggeman concept [22] deals with two polarizable spherical objects.)

The equation (16) gives effective conductivity for an isotropic composite. It contains four poles. So one can state that for this composite $\widetilde{\sigma}$ will be the same as one of principal components of the effective conductivity tensor in a system of equally orientated noninteracting spheroids of three (or four if percolation occurs) kinds. The depolarization factors of these spheroids, $s_{1}, s_{2}, s_{3}$, are related by the following expression:

$s_{1}^{\prime \prime}+2\left(s_{2}^{\prime \prime}+s_{3}^{\prime \prime}-1\right)=p_{2}$

The spectral density that corresponds to the equations (13) and (16) is of the form:

$$
g(t)=\sum_{n} B_{n} \delta\left(t-s_{n}\right)
$$

where $\delta\left(t-s_{n}\right)$ is Dirac delta function. However, as already noted, actually a continuous mode spectrum shows itself. It appears after averaging over macroscopic volume, so the poles in (19) are broadened. This means that in real practice it makes sense to deal with a generalization (19) that accounts for this broadening. One can formally do it by changing $\delta$-function for some (close to it) function $\chi(t)$ with a nonzero halfwidth $\Delta$. In this case, evidently, a passage to the limit $\lim _{\Delta \rightarrow 0} \chi(t, \Delta)=\delta(t)$ is to be obeyed. One of the simplest examples is Lorentz function

$$
\chi(t, \Delta)=\frac{\Delta}{\pi} \frac{1}{t^{2}+\Delta^{2}} .
$$

In this case integration in the expression (2) may be made explicitly. Setting $g(t)=\sum B_{n} \chi\left(t-s_{n}\right)$ and dropping out the mathematics, we give the final result (that is a generalization of expressions (13) and (16)):

$$
\begin{aligned}
& \frac{F(s)}{p_{1}}=\frac{B_{0}}{s}+\frac{\Delta}{\pi} \sum_{n=1}^{2(3)} \frac{B_{n}}{\left(s-s_{n}\right)^{2}+\Delta^{2}} \times \\
& \times\left\{\begin{array}{l}
\frac{s-s_{n}}{\Delta}\left(\operatorname{arctg} \frac{1-s_{n}}{\Delta}-\operatorname{arctg} \frac{s_{n}}{\Delta}\right)- \\
-\ln \left[\left(\frac{1}{s}-1\right) \sqrt{\left.\frac{s_{n}^{2}+\Delta^{2}}{\left(1-s_{n}\right)^{2}+\Delta^{2}}\right]}\right.
\end{array}\right\} .
\end{aligned}
$$

Here $B_{n}=B_{n}^{\prime}, s_{n}=s_{n}^{\prime}$ for generalization of expression (13) and $B_{n}=B_{n}{ }_{n}, s_{n}=s_{n}$ for generalization of expression (16). It should be also noted that $\Delta$ is not a new independent parameter since it may be determined from the normalization condition (3).

In conclusion let us make some remarks concerning applicability of the above approach. It is known that the effective conductivity is introduced when it is possible to consider an effective macroscopic (i.e., averaged over vo-lumes whose size greater than the characteristic size of nonuniformities) field. This condition becomes difficult to attain near the percolation threshold where the correlation length may become large and the system properties are determined by scaling invariance. The above approach by no means takes these things into account (though it seems possible that it can be generalized in the necessary way). On the other hand, we do not consider some size effects, such as the Fuchs effect (see $[23,24]$ ) or Sandomirsky quantum-size effect $[25,26]$ when a characteristic length (say, grain size) of a system becomes, respectively, of the order of charge carrier free path or de Broglie wavelength. Of course, electron scattering on interfaces (boundaries between phases) may be of importance (especially when taking into account dissipation). This effect, however, goes beyond the above consideration. If should be only noted that a formal account of size effects within the framework of our approach may be reduced to changing the bulk conductivity of a phase (phases) for some its modification.

\section{References}

1. D.J. Bergman, Analytical properties of the complex dielectric constant of a composite medium with application to the derivations of rigorous bounds and to percolation problems, in: Electrical Transport and Optical Properties of Inhomogeneous Media, AIP Conf. Proc. No 40, Eds. J.C. Garland and D.B. Tanner, pp.4661, AIP, New York (1978).

2. D.J. Bergman, The dielectric constants of a composite material - a problem in classical physics // Phys. Rep. C, 43(9), pp.377407 (1978).

3. J. Korringa, The influence of pore geometry on the dielectric dispersion of clean sandstones // Geophysics 49(10), pp.17601762 (1984).

4. D. Stroud, G.W. Milton and B.R. De, Analytical model for the dielectric response of brine-saturated rocks // Phys. Rev. B 34(8), pp.5145-5153 (1986).

5. K. Ghosh and R. Fuchs, Critical behavior in the dielectric properties of random self-similar composites // Phys. Rev B 44(14), pp.7330-7343 (1991).

6. M. Hornfeck, W. Theiss and R. Clasen, IR spectroscopy of porous silica gels // J. Non-Cryst. Sol. 145(1-3), pp.154-158 (1992).

7. R. Fuchs and F. Claro, Spectral representation for the polarizability of a collection of dielectric spheres // Phys. Rev. B 39(6), pp.38753878 (1989).

8. F. Claro and R. Fuchs, Collective surface modes in a fractal cluster of spheres // Phys. Rev. B 44(9), pp.4109-4116 (1991).

9. K. Hinsen and B.U. Felderhof, Dielectric constant of uniform spheres // Phys. Rev. B 46 (20), pp.12955-12963 (1992).

10. R. Stognienko, Th. Henning and V. Ossenkopf, Optical properties of coagulated particles // Astron. Astrophys. 296, pp.797809 (1995).

11. R. Fuchs, R.G. Barrera and G.L Carrillo, Spectral representations of the electron energy loss in composite media // Phys. Rev. B 54(18), pp.12824-12834 (1996).

12. A.G. Belous, A.V. Goncharenko, V.R. Romaniuk and E.F. Venger, Low-frequency dielectric function of close-packed composites // Proc. SPIE 2863, pp.141-151 (1996).

13. A.R. Day and M.F. Thorpe, The spectral function of composites: the inverse problem // J. Phys.: Condens. Matter 11(12), pp.25512568 (1999).

14. K. Ghosh and R. Fuchs, Spectral theory for two-component porous media // Phys. Rev. B 38(8), pp.5222-5236 (1988).

15. J. Monecke, Microstructure dependence of material properties of composites // Phys. stat. sol. (b) 154, pp.805-813 (1989). 


\section{A.V. Goncharenko: Conductivity of a two-phase composite: An approach...}

16. J. Monecke, Bergman spectral representation of a simple expression for the dielectric response of a symmetric two-component composite // J. Phys.: Condens. Matter 6, pp.907-912 (1994).

17. Z. Hashin and S. Shtrikman, A variational approach to the theory of the effective magnetic permeability of multiphase material // J. Appl. Phys. 33(10), pp.3125-3131 (1962).

18. D.J. Bergman, Exactly solvable microscopic geometries and rigorous bounds for the complex dielectric constant of a two-component composite material // Phys. Rev. Lett. 44(19), pp.12851287 (1980).

19. G.W. Milton, Bounds on the complex dielectric constant of a composite material // Appl. Phys. Lett. 37(3), pp.300-302 (1980).

20. D.J. Bergman, Rigorous bounds for the complex dielectric constants of a two-component composite // Ann. Phys. 138(1) pp.78-114 (1982).
21. L.D. Landau and E.M. Lifshitz. Electrodynamics of Continuous Media, Nauka, Moscow, 1982 (in Russian).

22. D.A.G. Bruggeman, Berechnung verschiedener physikalischer Konstanten von heterogenen Substanzen. // Ann. Phys. 24(8), pp.636-679 (1935).

23. A.F. Mayadas, M. Shatzkes and J.F. Janak, Electrical resistivity model for polycrystalline films: the case of specular reflection at external surfaces // Appl. Phys. Lett. 14(11), pp.345-347 (1969).

24. A.F. Mayadas and M. Shatzkes, Electrical-resistivity model for polycrystalline films: the case of arbitrary reflection at external surfaces // Phys. Rev. B 1(4), pp.1382-1389 (1970).

25. V.B. Sandomirsky, Quantum-size effect in a semimetal film // ZhETP 25(1), pp.162-166 (1967) (in Russian).

26. M. Mudrik, S.S. Cohen and N. Croitoru, Electron conductivity of very thin metal films // Thin Solid Films 226 (1), pp.140-143 (1993). 